\title{
Acceptability of UC781 Gel as a Rectal Microbicide Among HIV-Uninfected Women and Men
}

\author{
Ana Ventuneac · Alex Carballo-Diéguez - Ian McGowan · Robert Dennis · \\ Amy Adler · Elena Khanukhova - Charles Price · Terry Saunders • \\ Chomchay Siboliban · Peter Anton
}

Published online: 11 September 2009

(C) The Author(s) 2009. This article is published with open access at Springerlink.com

\begin{abstract}
We studied the overall acceptability of UC781 gel formulation when applied rectally. Ten women and twenty-six men, all HIV-uninfected, were enrolled in a Phase 1, randomized, blinded, placebo-controlled safety and acceptability study of the vaginal microbicide gel UC781 applied rectally. Participants were randomized to three groups: $0.1 \%$ UC781 gel, $0.25 \%$ UC781 gel, or a placebo gel. Acceptability was assessed using structured questionnaires and qualitative in-depth interviews. After using UC781 gel rectally for seven consecutive days, participants' reports suggest that a UC781 gel formulation is highly acceptable and comparable to a placebo gel. The gels received favorable ratings overall and on attributes such as color, smell and consistency. All of the participants reported high intentions to use a gel like the one they used in this study. Acceptability research is essential in early phases of microbicide development to identify potential problems, understand user preferences, and introduce changes if needed.
\end{abstract}

\footnotetext{
A. Ventuneac $(\bowtie) \cdot$ A. Carballo-Diéguez

HIV Center for Clinical and Behavioral Studies, New York State

Psychiatric Institute and Columbia University, 1051 Riverside

Drive, Unit 15, New York, NY 10032, USA

e-mail: ventune@pi.cpmc.columbia.edu

\section{McGowan}

Magee-Womens Research Institute, Pittsburgh, PA, USA

R. Dennis · E. Khanukhova - C. Price - T. Saunders ·

C. Siboliban · P. Anton

Center for HIV Prevention Research at David Geffen School

of Medicine at UCLA, Los Angeles, CA, USA
}

\section{A. Adler}

The Translational Science Corp., Mill Valley, CA, USA
Keywords Microbicide acceptability .

Rectal microbicides - Anal intercourse - UC781 · HIV prevention

\section{Introduction}

Microbicides are compounds that may reduce the risk of HIV transmission during sexual intercourse and, if found effective, may have a great impact on slowing the HIV epidemic [1-3]. Both vaginal and rectal topical microbicides are currently under development, with the former being in more advanced stage of testing [4]. Based on evidence that showed up-take among gay men of lubricants containing Noxoxynol-9 (N-9), a detergent-based spermicide, before it was actually tested for both safety in the rectal compartment and efficacy in preventing HIV [5], it is generally assumed that if a vaginal microbicide were shown to be effective, it will be used rectally by women and men who engage in receptive anal intercourse (RAI). Given that previous research has documented that RAI is a common practice in a significant proportion of the US population [6-18] and that it is estimated to be riskier than other sexual practices including vaginal intercourse [19-21], testing products developed for vaginal use for their safety in the rectal compartment is crucial [4].

To prevent HIV transmission, microbicides need not only to be efficacious and safe for people to use, but also need to be used correctly and consistently by individuals at risk for acquiring HIV [1, 2, 22-27]. The latter issue has been referred to as acceptability, defined as "the voluntary sustained use of a method in the context of alternatives" (p. 47) [27].

To inform the development of products that fit people's needs, past research on rectal microbicide acceptability has 
mainly involved surveys of the attitudes and preferences of men who have sex with men (MSM) about hypothetical and surrogate products (without active agents) [1, 5, 22, 23, 28-30]. Typically, participants were informed about a hypothetical product (level of protection provided by condoms was sometimes presented as an anchor) and asked their preferences about various product characteristics and willingness to use the product in the future. In one such study with a sample of 307 Latino MSM in NYC, $92 \%$ of the men indicated that they would use a lubricant that could protect against HIV transmission [1]. Studies have found that willingness to use a hypothetical product was associated with unprotected RAI, dislike for condoms, and selfefficacy for product use [28-30]. Recognizing that there may be variation in the formulation and amount of the delivery vehicles that will carry an efficacious agent and the amount of volume needed to confer protection, Carballo-Diéguez and colleagues using surrogates as study products, found that a gel is preferred over a suppository and in volumes of up to $35 \mathrm{~mL}[22,23]$ Among a small sample of women, Exner and colleagues found that most women were interested in a rectal microbicide [24].

To date, only one Phase 1 trial evaluated the safety and acceptability of a product administered rectally, Advantage 24 , a gel containing N-9 considered a potential microbicide at that time $[25,31,32]$. Gross and colleagues found that acceptability was low among the sample of monogamous, HIV-seroconcordant male couples; less than half reported intentions to use it in the future if it were approved for rectal use [25]. Participants reported rectal fullness and discomfort with most of the complaints involving insertion of the applicator, its sharp edge at the tip, and dispensing of air with the gel. Other complaints involved comments about the gel being too sticky and drying out too quickly; having an unappealing taste, smell, and color. Furthermore, although the initial studies did not identify safety problems [31], subsequent studies concluded that N-9 causes epithelial disruption increasing susceptibility to HIV infection and thus, placing individuals at increased risk for contracting HIV [33, 34].

Based on prior research, several factors have been identified as salient and appropriate in assessing acceptability comprehensively at each phase of product development [26]. For Phase 1 trials, those factors are: (1) formulation-associated variables, such as texture and viscosity, product scent, color, and taste, and desirable/ appealing elements of formulation; (2) application-associated variables, such as ability to adhere to instructions, ease of product preparation (e.g., filling applicator with product), ease of application, portability, applicator design and disposal preferences; (3) use-associated variables, such as frequency and timing of product use, partner-specific issues, lubrication, product consistency post-use, desirable/ appealing elements of use, use with/without condom, changes in hygiene practices secondary to use, and changes in sexual pleasure secondary to use; and (4) related covariates, such as history of vaginal and anal product use, frequency of vaginal and anal sex, relationship "harmony"; relationship communication. Assessing acceptability together with determining the clinical safety profile of products is recognized as crucial in early phases of microbicide trials $[22,23,26]$. Lack of acceptability of a product in early stages of development would raise an important alert for scientists to retool the product, its delivery system or mode of use before embarking on larger and more expensive efficacy studies.

In this Phase 1 randomized, blinded, placebo-controlled trial, we evaluated the vaginal microbicide candidate, UC781 (CONRAD, Arlington, VA), a non-nucleoside reverse transcriptase inhibitor (NNRTI) of the HIV-1 reverse transcriptase (RT) enzyme [35], when administered rectally as a gel in HIV-1 uninfected adults [36]. In this report, we compare participants' product-use ratings in three treatment groups $(0.1 \%$ UC781 gel, $0.25 \%$ UC781 gel, or a placebo gel) to examine what factors contribute to or detract from acceptability of UC781 applied rectally among women and men. Our hypothesis was that after one week of practice in the use of the product, respondents would, on average, report high intentionality, defined as an average score in the upper one-third of the Likert scale, to use the product in the future every time they have receptive anal intercourse. Furthermore, participants would report high intentionality to use the product on occasions when they do not use condoms. Additionally, we anticipated that, although many of the individuals who voluntarily enroll in a study like ours would be predisposed to like the product (as measured at baseline), the experience of using the gel would on average increase their intentionality ratings. We did not anticipate differences among the three groups in intentionality to use the product in the future, since the placebo used in this study from the investigator's point of view, was quite similar to the formulation for the UC781 gel.

\section{Methods}

\section{Participants}

A Phase 1 randomized, blinded, placebo-controlled trial was conducted at the Center for HIV Prevention Research at David Geffen School of Medicine at UCLA, Los Angeles, CA, USA, between December 2007 and April 2008. HIVuninfected women and men were recruited using four strategies: (1) clinician-patient referrals; (2) use of existing study registries that contain contact information of individuals who gave informed consent to be reached for future 
studies; (3) participant referrals (participants refer their friends or partners who may meet eligibility criteria); and (4) study advertisements. Individuals were eligible to participate if they met the following criteria: (1) 18 years of age or older; (2) reported a history of consensual RAI at least once in lifetime; (3) received a negative HIV antibody test and STI tests (for N. gonorrhea, C. trachomatis, syphilis and active HSV disease) at screening; (4) being in good health as assessed by a physical examination, and not meeting any exclusionary criteria (e.g., history of significant gastrointestinal bleeding, allergies to methylparaben, propylparaben, sorbic acid, use of certain medications); and (5) not having a history of alcoholism or injection drug use. Participants also had to be willing to abstain from insertion of anything per rectum other than the study gel, including abstaining from RAI, 1 week prior to treatment, 1 week prior and after each flexible sigmoidoscopy, as well as abstain from chronic use of aspirin and non-steroidal antiinflammatory drugs (e.g., Advil). In addition, women had to receive a negative pregnancy test, not breastfeed, and be post-menopausal or use an acceptable form of contraception (e.g., barrier method, IUD, hormonal contraception, surgical sterilization, or vasectomization of male partner).

\section{Procedures}

A detailed description of study procedures as they pertain to the safety aim of the study exceeds the scope of this publication and will be presented elsewhere [36]. In terms of the behavioral evaluations, individuals who were deemed eligible after the screening period took part in a structured assessment, the Baseline Behavioral Questionnaire administered by Web-based, Computer Assisted SelfInterview (CASI). Participants were given the option of completing the questionnaires over the Internet at any location convenient to them. Those without access to a computer or the Internet completed the questionnaires at the study site. Participants were provided with assistance, as needed.

A total of $36 \mathrm{HIV}$-uninfected participants (10 women and 26 men) were randomized to one of three groups: $0.1 \%$ UC781 gel, $0.25 \%$ UC781 gel, or a placebo gel (12 per group). UC781 $0.1 \%$ and UC781 $0.25 \%$ gels are off-white to light yellow aqueous formulations containing, respectively, 0.1 or $0.25 \%(\mathrm{w} / \mathrm{w})$ UC781, Carbomer 974P, methylcellulose, glycerin, purified water and common preservatives (methylparaben and propylparaben). Both 0.1 and $0.25 \%$ UC781 strength gels were adjusted to $\mathrm{pH} 5.2$. The placebo gel, hydroxyethyl cellulose (HEC), is a clear aqueous gel formulation containing non-active substances: sodium chloride, hydroxyethyl cellulose, sorbic acid, sodium hydroxide and purified water. All three gels were similar to the touch for investigators, although viscosity differed markedly. The volume of each dose was $3.5 \mathrm{~g}$ (approximately $3.5 \mathrm{~mL}$ ).

Study gels were packaged in an overwrapped, single dose, prefilled vaginal applicator that had been designed and FDA-approved for use in the vaginal microbicide clinical trials of UC781 [37]; this was not a rectal-specific applicator design. The single-use vaginal applicator consists of a barrel and plunger with a screw-on cap [38]. The barrel measures $114 \mathrm{~mm}$ long by $12.7 \mathrm{~mm}$ wide with a tapered and rounded tip and a flanged base. Applicators were labeled with "Caution: New Drug-Limited by Federal (United States) law to investigational use." Seven applicators were dispensed in a single box that included an instructional pamphlet with diagrams to depict proper insertion. A single, blinded label was affixed to each box with the following information: randomization number, protocol number, storage conditions and dosing instructions. Only the investigational pharmacy was unblinded to group throughout the study.

Once randomized, study staff administered a single dose of the study gel after a preparatory Normosol-R pH 7.4 enema. Participants had the option of lying on their side, flat on their back with knees bended, or standing and bending over. After a 7-day wash-out period, study staff reviewed the instructions with participants before dispensing the 7-day supply for home use. Participants selfadministered once daily outpatient doses of the study gel for 7 days and then completed a Product Acceptability Questionnaire via Web-based CASI. After completing all study procedures (three separate flexible sigmoidoscopies and multiple other mucosal irritation assessments), participants took part in a semi-structured phone interview conducted by a gender-matched interviewer (either a male interviewer for a male participant, or a female interviewer for a female participant) with experience in rectal microbicide research. On average, the interviews lasted $35 \mathrm{~min}$.

Participants completed six study visits and were compensated a total of $\$ 750$ in cash for their time. To encourage completion of the trial, participants were paid more toward the end of the trial. No compensation was provided for the eligibility screening.

\section{Measures}

Questionnaires were posted on a Web page accessible to study participants with a username and password. The purpose of using this assessment method was to accommodate participants' needs and, at the same time, to encourage them to be as honest as possible in their reporting, minimizing a need to conform to an interviewer's expectations in a face-to-face encounter. To minimize missing data due to browsing from screen to screen too quickly, a "Prefer not to answer" option was 
presented for every questionnaire item to identify purposely skipped questions. All participants completed the Web-based questionnaires.

\section{Baseline Behavioral Questionnaire}

Sexual Behavior Participants were asked to report their sexual behavior during the previous two months, including number of sexual partners and unprotected RAI occasions. Questions were posed both in formal and vernacular language to increase comprehension.

Rectal Microbicide Intentions At baseline, we asked: "If a rectal microbicide were available that provided some protection against HIV, and it were presented as a gel, how likely would you be to use it every time you have anal intercourse?" The response scale ranged from $1=$ "Extremely unlikely" to $10=$ "Extremely likely".

\section{Product Acceptability Questionnaire}

The acceptability assessment, completed once participants finished using the study product, included a general question ("Overall, -i.e., considering all the episodes in which you used this gel—how much did you like the product?"); it also included specific questions on the level of like/dislike of the products' physical properties, application process, and applicator. Participants responded to these questions on a 10-point Likert scale $(1=$ "Disliked very much" to $10=$ "Liked very much"). They were asked specifically about how the product felt inside their rectum ("How much did you like how the gel felt inside your rectum [immediately after inserting it/30 $\mathrm{min}$ after inserting it]?") Given that participants were asked to refrain from engaging in receptive anal intercourse during the study, we did not assess product-use acceptability in the context of sexual intercourse. However, participants were presented hypothetical situations such as, "If a gel were available that contributed to provide some protection against HIV, and it looked like the one you have used in this study, how likely would you be to use it every time you have anal intercourse?", "How likely would you be to use a gel that contributed to provide some protection against HIV on the occasions when you don't use condoms?", and "How likely would you be to use a gel if you had to wait 30 min after application before having intercourse?", as well as "How much would you be willing to spend on a gel per sexual occasion?" $(1=$ "About as much as one spends on condoms" to $3=$ "Three times as much"). Participants were also asked whether they had used a volume different than the one recommended (each pre-filled applicator contained $3.5 \mathrm{~mL}$ of gel), and if any problems were experienced (e.g., leakage, soiling of underwear or linens); for those experiencing problems, we asked how bothersome they were ( $1=$ "Not at all" to $10=$ "Very much"). Finally, participants were asked about their willingness to use a higher volume and whether they had any product recommendations.

\section{Semi-Structured Interview}

After reviewing the responses the participants provided to the Web-based CASI, gender-matched interviewers conducted a phone interview with each participant during which the specific circumstances of product use for the "home assignment" were explored. Interviewers sought to clarify any discrepancies that might have been observed in the reported quantitative data, as well as to contextualize the quantitative reports. All interviews were audio recorded and transcribed.

\section{Data Analysis}

To account for non-normal data, Kruskal-Wallis nonparametric tests were used to evaluate whether the ratings were significantly different by group $(0.1 \%$ UC781 gel, $0.25 \%$ UC781 gel, or a placebo gel). The qualitative data were coded based on the structure of the interview guide that covered topics such as opinions about the gel, applicator, administration process, and use-associated variables. Coded passages were summarized and quotes that illustrated specific topics were selected to complement the quantitative data.

\section{Results}

\section{Sample Description}

Ten women and 26 men completed the trial. Participants ranged in age from 24 to 64 years $(M=41.70$, $\mathrm{SD}=10.99$ ). Three-quarters completed a high school education or higher; half were employed either full or parttime, having a median annual income in the \$10,001$\$ 20,000$ range; nearly two-thirds identified as being of ethnic minority background (14 participants identified as African-American, 5 as Latino, 1 as Asian or Pacific Islander, and 1 as Native American; 14 identified as White or European American); and over three-quarters of the men identified as gay and nearly three-quarters of the women identified as straight.

\section{Sexual Behavior}

Among the male participants, one completed the wrong version of the sexual behavior questionnaire; data for this 
case were excluded from this description. At baseline, $72 \%$ (18 of 25) of the male participants reported having had at least one male sexual partner $(M d n=2.00)$ in the prior 2 months with whom they had engaged in anal intercourse. Forty percent ( $n=10$ ) of the participants reported having engaged in RAI and 60\% $(n=15)$ in insertive anal intercourse in the previous 2 months. On average, participants reported having $4.72(M d n=1.00)$ occasions of RAI in the previous two months, slightly more than half $(55.1 \%)$ of them unprotected. Slightly more than three-quarters $(78.7 \%)$ of the insertive anal intercourse occasions $(M d n=1.00, M=14.37, \mathrm{SD}=45.48)$ in the previous 2 months were unprotected.

Half of the male participants who reported having had at least one female sexual partner in the previous 2 months ( $n=10 ; M=1.42, \mathrm{SD}=2.70)$, reported engaging in anal intercourse with their female partners. All but one of the ten anal intercourse occasions reported were unprotected. Similarly, the majority of the total vaginal intercourse occasions were unprotected.

Among the female participants, 5 of the 10 women reported having had at least one male sexual partner $(M d n=1.00)$ in the previous 2 months. Three reported engaging in anal intercourse; half of the total anal intercourse occasions reported (10) were unprotected.

\section{Product Acceptability}

Kruskal-Wallis non-parametric tests were used to evaluate whether the ratings were significantly different by condition (Table 1). As expected since the study gels were similar to the touch, our findings showed that there were no statistically significant group differences in the acceptability ratings of the two UC781 (0.1 and $0.25 \%)$ gels as compared to the placebo gel. Similar ratings were observed between women and men. Because of concerns of insufficient statistical power to detect differences and in order to get an estimate of the magnitude of the effect, we examined eta-squared $\left(\eta^{2}\right)$ from the ANOVAs (see Table 1) to get a measure of the relationship between each acceptability variable (dependent variable) and the treatment group (independent variable). Eta-squared is the proportion of variance that is attributable to the treatment condition. For example, ratings for being bothered by soiling of underwear or linens were higher among the three men who reported soiling in the placebo group $(M=4.00$, $\mathrm{SD}=1.00)$ as compared to the four men in the $0.1 \%$ UC781 group $(M=2.50, \mathrm{SD}=1.29)$. However, because of the small sample size in each group, this test was not statistically significant $\left(\eta^{2}=.529\right)$.

For descriptive purposes, we combined results across the three gel conditions to describe overall acceptability and ratings on formulation, application, and use.

\section{Overall Product Ratings}

Overall, the majority of participants $(56 \% ; 20$ out of 36 ; $95 \%$ confidence interval $(\mathrm{CI}), .40-.71)$ reported liking the product used in this study (ratings were in the upper third of the 10-point scale), irrespective of condition. The remainder of the sample felt neutral, indicating that overall, they neither disliked nor liked the product. During the semi-structured interview, one participant explained his reasons for a high rating:

I felt that the product was more practical than lubricants that I'd been using in the past because it wasn't as messy, it didn't stick to my fingers, it wasn't oily... and could be applied directly. (ID 452, $0.1 \%$ UC781 gel, male)

It is important to note that participants rated the product after they had had the opportunity to use it repeatedly. Familiarity with the product, applicator, and mode of administration gained in successive days may have had a positive effect on the final rating.

[At the beginning] I was making myself nervous doing it. "I have to get it out of the box, open it, plunger in, make sure I do it right, make sure I don't let any [gel] come out." ... I got more comfortable with it over the days... [In the end,] it took a little over a minute. (ID 444, 0.25\% UC781 gel, male)

\section{Formulation-Associated Factors}

Participants reported liking the product's color $(62 \% ; 21$ out of 34 who provided a rating; 95\% CI, .45-.77), smell $(55 \%$; 6 out of $11 ; 95 \%$ CI, .27-.80), taste $(57 \%$; 4 out of 7 ; $95 \% \mathrm{CI}, .23-.86)$, and consistency $(61 \% ; 22$ of $36 ; 95 \% \mathrm{CI}$, .44-.75). Only one male participant indicated disliking both the color and consistency of the study product (placebo gel). Another male participant (0.1\% UC781 gel) reported disliking its consistency and a female participant disliked its color $(0.1 \%$ UC781 gel). The remaining participants felt neutral, indicating they neither disliked nor liked the product's attributes.

In the semi-structured interview, some participants further described what they liked about the product they tried:

I never felt it on my hands, I never felt greasy, I never felt really the residue of it. (ID 408, $0.1 \%$ UC781 gel, male)

Even days after I put it in there, I didn't get no reaction, no allergy reaction, and I'm from [name of island]. I get allergy rashes from the water here. But I didn't get any kind of reaction at all from the gel. (ID 439, $0.1 \%$ UC781 gel, male) 


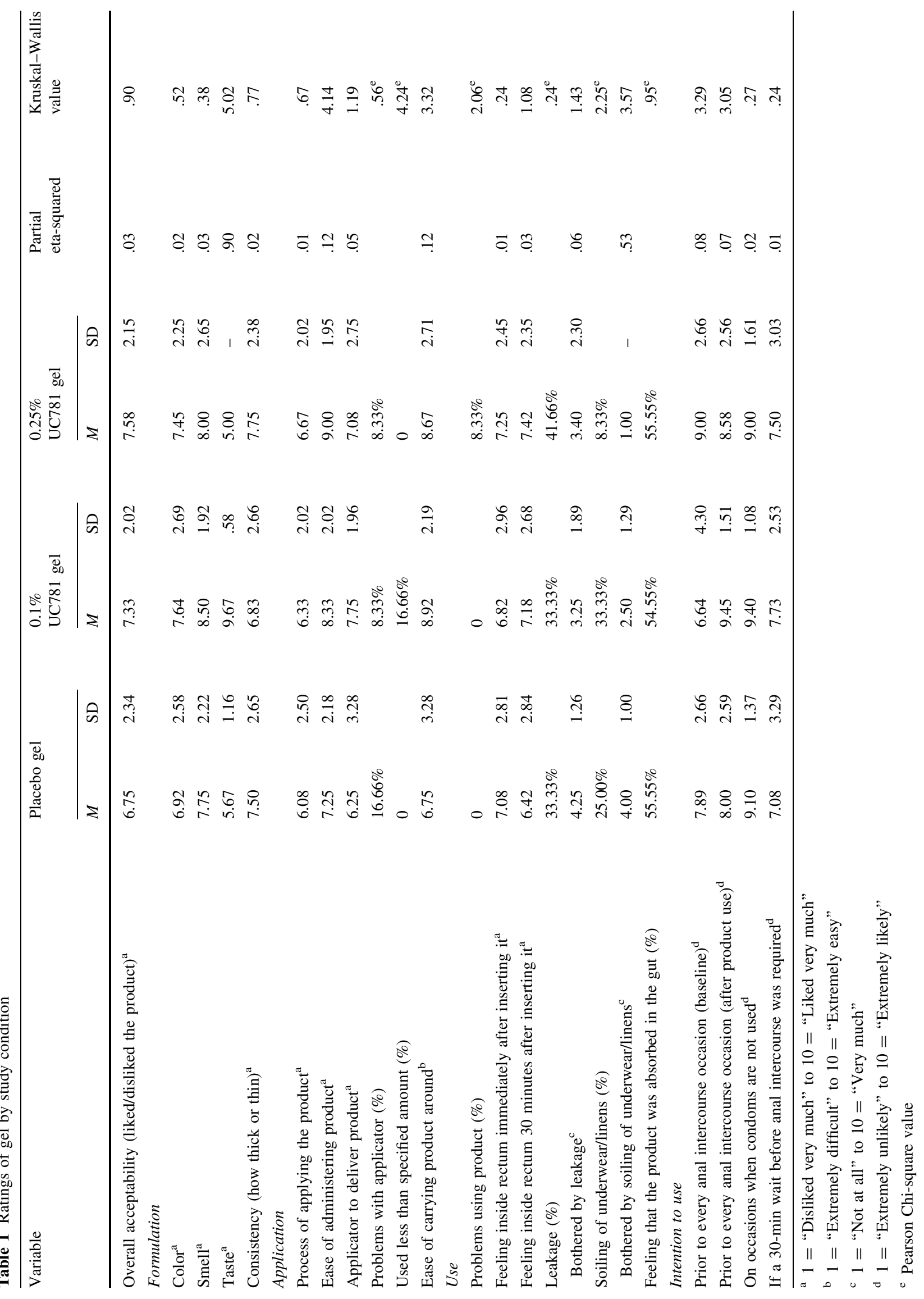


Others reported that, initially, the gel felt cold (ID 424, $0.1 \%$ UC781 gel, female) or that it burned (ID 410, 0.1\% UC781 gel, female), but the sensations disappeared when the participants became familiar with the product.

The first time I tried it, I had a little bit of ardor. It was stingy a little too much. But I think it was my... I had had some hot peppers for dinner, so I wasn't sure which was causing that... After that day, I never had that problem anymore. (ID 451, placebo gel, male)

\section{Application-Associated Factors}

Fewer participants rated the process of applying the study product favorably $(36 \% ; 13$ out of 36 ; $95 \% \mathrm{CI}, .22-.53)$; the majority felt neutral about it (neither disliked it nor liked). Seventy-two percent (95\% CI, .55-.84) rated dispensing the product as very easy; only one participant ( $0.25 \%$ UC781 gel) reported it as difficult. Although the average rating for the applicator was in the upper third of the scale $(58 \% ; 21$ out of 36 ; $95 \%$ CI, .42-.73), four participants ( 1 woman and 3 men) reported disliking it. Four male participants indicated that it would be difficult to carry the product applicator around. Eighty-one percent (95\% CI, .65-.91) favored a disposable product, such as the one used in the study, over a refillable one.

During the semi-structured interviews, some participants stated that they had no complaint at all about the applicator or the application process, "It's compact, and portable, and easy enough to use" (ID 418, 0.1\% UC781 gel, male). However, several others explained that they experienced problems using rectally an applicator that was designed for vaginal use. The vaginal applicator elicited complaints even after a lubricant was used to facilitate insertion:

[U]sually I only do enemas occasionally you know, just for general health purposes, to keep my inside clean. [...] I don't use the lubricant with that [tip of enema delivery system], cause it's too small and it's really smooth and just hasn't presented any problems. But with the gel for the study I definitely needed to use a lubricant because that was- just the tip of the applicator is a little big. [...] You know, it would be uncomfortable without it [lubricant]. (ID 405, 0.25\% UC781 gel, female)

The only problem I had with it is the applicator itself because the top is open you know. And, when you put it in, it like hurts the walls when you're inserting it. My suggestion that I told the doctor that if they made it more round, like Tampax applicators are and make it easier for it to go in. (ID 427, 0.25\% UC781 gel, female)

Other problems with the applicator included difficulty in handling it while trying to insert the study product and not having tactile indicators to signal both when the applicator was sufficiently inserted in the rectum and that all of the gel was ejected.

I could see some dudes kind of drunk or high or whatever shoving the whole thing up there, and then you have to worry about getting it out. [You need to] have like a grip mark on it and that's where your fingers go, and above that is how far it's supposed to go up your butt. (ID 455, placebo gel, male)

For one participant, applicator-related problems resulted in using less than the specified amount of gel on two occasions.

When I was pushing on the thing, $[\ldots]$ whatever the name of those two pieces of plastic [is], the long skinny piece [plunger] through the other piece [barrel], I couldn't tell if it was completely at the end. [...] The thing [applicator] wasn't big enough to hold on to and to push in on it....I found the applicators a little cumbersome. It was a little wide and it seemed a little hard to push the... It seems as if it needs a little thing on the end where you pose your fingers on. (ID 408, $0.1 \%$ UC781 gel, male)

Having to unwrap and assemble the applicator were other complications mentioned by participants, particularly in the initial use of the study product at home.

I realize they have to keep it fresh and unopened, but I couldn't get the package... I had to use my fingernail clippers a few times to open it. (ID 408, 0.1\% UC781 gel, male)

I had a hard time the first time. I forgot everything that the staff told me. So the next day, I took off the little cover [cap] and put the syringe [plunger] in, and then some gel popped out. But I shoved it in there anyway. ... The next day, I read the paper again, and they said "leave the blue cap on, then put the plunger until you hear a click, and then take the cap off." (ID 439, $0.1 \%$ UC781 gel, male)

Concerning the position when applying the product, some participants felt more comfortable doing it while standing up while others preferred to be lying down, on their side, or squatting.

My first time I tried it I was standing... It was difficult. The second time, I was lying down and it was much easier. And by the third time, I was almost a pro. (ID 430, 0.1\% UC781 gel, female)

I was lying down on my bed and entered it with my legs up in the air. I think a little bit fell out because I wasn't really sure how I was going to do it. [Then I said,] 'I'm going to do it in a squatting position 
because none of it slipped out that time.' And I continued to do it in a squatting position all the way through. (ID 432, placebo gel, female)

Participants indicated that any initial problems they experienced with applying the study product were overcome with practice.

It became easier and easier to do it [insert the product] and then I actually [could] do it in a public restroom. (ID 439, 0.1\% UC781 gel, male)

\section{Use-Associated Factors}

Participants rated the study product favorably on its feeling in the rectum immediately after insertion $(54 \% ; 19$ out of 35; 95\% CI, .38-.70) and/or $30 \mathrm{~min}$ after insertion (49\%; 17 out of 35 ; 95\% CI, .33-.65). Of the remaining participants, all but two felt neutral about how the study gel felt inside the rectum after insertion and after $30 \mathrm{~min}$ had passed. One participant disliked how the placebo felt inside the rectum after insertion and after $30 \mathrm{~min}$ had passed. In addition, one participant disliked how $0.1 \%$ UC781 felt after $30 \mathrm{~min}$ passed. The majority (across the three conditions) felt that the study product was absorbed in their gut (55\%; 16 out of $29 ; 95 \%$ CI, .37-.72). Some leakage was reported by $36 \%$ of the sample across the three conditions (13 out of $36 ; 95 \%$ CI, .22-.53) but they were bothered very little by it. Soiling of underwear and linens was reported by a little over a fifth of the sample ( 8 out of 36 ; $95 \%$ CI, .11-.38).

In the semi-structured interview, participants explained,

Before I used it, I thought, "Oh, this is going to be a big mess, it's going to be goopy everywhere; it's gonna take a while to get rid of it." And I used it [and] it was a very easy process. A couple of times [there was] leaking, but the leaking seemed very liquidy, as compared to gooey. It's the reason that I thought if it [were] thicker, maybe it wouldn't do that. (ID 408, 0.1\% UC781 gel, male)

When I would put it in, I don't know whether it was my nerves or whether it was the gel, but I would get the sensation that there was something in my butt and I would feel like pushing. I would get a lot of flatulence and I would feel something come out, but I don't know if it was specifically the gel. (ID 444, $0.25 \%$ UC781 gel, male)

By contrast, another participant stated,

It did not feel any different than using just regular lube, of K-Y. (ID 422, 0.1\% UC781 gel, male).

\section{Recommendations}

Most of the product recommendations were related to the applicator. In this study, a vaginal applicator was used to administer the study gel rectally. Suggestions included making the applicator smaller, making the tip thinner and softer specifically for rectal use, changing the tip to reduce the chance of accidental poking of rectal wall, making it easier to grip and requiring no user-assembly (inserting the plunger in the barrel), and adding a small packet with lubricant in the package to help with insertion. A few participants suggested making the gel thicker and less runny ("it should be the consistency of cold pie filling or cold Cool Whip"), and adding various flavors (none were suggested). A compact ("just like condoms"), stylish and appealing ("not medical appearing"), and "eco-friendly and biodegradable package" was suggested. Participants stated,

Carrying it becomes difficult, especially if you are a man. What are you gonna do? Put it in your wallet? ... It is much easier to carry a condom than that. (ID 420, $0.25 \%$ UC781, male).

[The applicator] is kind of noticeable. ... There is still stigma if people could tell you had a condom in your pocket. "This is a big slut," written across your forehead (ID 444, $0.25 \%$ UC781 gel, male).

\section{Intention to Use Gel in the Future}

As expected, after participants used the study product, there was no statistically significant difference in intentionality to use the product in the future among the three groups. All of the participants rated their intention in the upper third of the Likert scale ("extremely likely") after they had a chance to use the study product. Although not statistically significant (Wilcoxon Signed-Rank Test $z=1.09, P=.28$ ), intention to use the gel in the future increased with respect to the intention reported prior to any rectal experience with the gel (baseline: $M=7.88$, $\mathrm{SD}=3.55$; post-trial: $M=8.66, \mathrm{SD}=2.31$ ). Participants reported high intentions to use a gel on occasions when condoms are not used and if a 30-min wait time was required for a microbicide to take effect before they could engage in anal intercourse.

In the semi-structured interviews, some people qualified their enthusiasm for the product: "It would be easy [to use] but it probably depends on the heat of the moment. Ain't nobody always stop and do that" (ID 410, $0.1 \%$ UC781 gel, female). When asked about having to wait $30 \mathrm{~min}$ after application before having sex, a participant said, "If you have to put any time limit, it doesn't work as well" (ID 425, 0.1\% UC781 gel, male). 
When asked about using a product with sexual partners, participants did not consider it an issue:

Anything that would prevent something or ... creates a positive environment for sex would be all, they'd [referring to female partners] be all over it (ID 425, $0.1 \%$ UC781 gel, male).

I know he will like it, especially if it will prevent disease (ID 420, 0.25\% UC781 gel, male).

Using a lubricant would be way easier than putting on something that you don't really feel comfortable with (ID 417, placebo gel, male).

Almost all of the participants would be willing to use a microbicide even if half or twice as much as used in this study were required. The majority would be willing to spend as much as one spends for condoms; $25 \%$ would be willing to spend twice as much as condoms.

\section{Discussion}

This is one of the first studies in which a microbicide candidate, formulated and designed for vaginal use, was used rectally on several occasions by an ethnically diverse sample of HIV-uninfected women and men who reported at least one occasion of RAI in their lifetime. Given that the majority of participants reported having had anal intercourse, frequently unprotected, in the 2 months prior to the interview, our sample appears to be drawn from the population most likely to benefit from the future availability of an effective rectal microbicide. After using UC781 gel rectally for 7 days, all of the participants reported high intentions to use a product like the one used in this study in the future. Participants' reports suggest that this gel formulation of UC781 is highly acceptable and comparable to a placebo gel. The product was rated high by a majority of participants overall and on attributes such as color, smell, and consistency. Only two participants reported using less than the specified amount and a few reported being bothered by leakage and soiling of their underwear or linens. Considering together the different acceptability aspects assessed, this study by both women and men suggests that rectal use of a gel formulation of UC781 would be acceptable.

In the semi-structured interviews it was elucidated that people monitor themselves closely when they start using a product, attributing discomfort to the product rather than alternative sources (e.g., having eaten hot peppers). In the initial use of the study product, participants were concerned about administering it as instructed and using the required dose, as well as how the product felt (greasy, gooey) or behaved (the product is liquid and, therefore, leaks). It was not until participants had several tries at using the study product that they felt comfortable with it, which points out the importance of encouraging repeated use before eliciting opinions (unless participants find the product completely unacceptable on their first trial, in which case they should not be required to continue using it). More data are needed to determine the number of tries it may take individuals, on average, to feel comfortable inserting and dispensing a product.

From a methodological point of view, the combined use of quantitative and qualitative assessment tools proved quite rich. We were able to establish that although participants felt positive or neutral in their quantitative ratings of the application process and the applicator, when given the opportunity to elaborate, during the qualitative interviews, they pointed out a number of ways in which the applicator and its wrapper could be improved. This is useful information to take into account for the design of a rectal-specific applicator as well as to anticipate (and correct) situations that may interfere with adherence not just in Phase 1 clinical trials but also in larger Phase 2 or 3 trials. A complicated issue that needs attention is portability: Individuals who may want to take the product with them are likely to prefer a non-conspicuous presentation, something difficult to achieve if an applicator is required for delivery of the microbicide intrarectally. The appearance of the applicator used in this study is one intended for vaginal use, similar to that of a tampon and therefore participants may have had, a priori, negative associations for its use.

While our findings offer important acceptability data about this microbicide candidate, there are several study limitations that need to be noted. First, because this study was a clinical safety trial with a small sample size and specific eligibility criteria, data may not be generalizable to other populations. In addition, participants were volunteers who may differ in untold ways from the general population who may use (or not) the product in the future. Also, participants were asked to use the product at home for only 7 days; acceptability could be different if the product were used over a longer period of time. Participants may be responding to social desirability in their responses to questionnaires. Furthermore, participants did not try the product in the context of anal sex. Therefore, we are unaware how sexual pleasure or partners' reactions, including any penile reactions or symptoms, may affect the acceptability of a product.

We assessed acceptability in the context of a clinical trial that involved a specific dosing regimen (product had to be used once daily for 7 days) and other restrictions (many inclusion and exclusion criteria, and refraining from anal intercourse during the study) that did not represent "real world" use. Nevertheless, a microbicide formulated as a gel has been thought to be amenable particularly to gay men because it could be easily incorporated into routines and practices that already accompany anal sex $[5,22,23,39$, 
40]. Prior research indicates that most MSM use lubricants and douches or enemas in preparation for anal intercourse $[1,5,40]$. Although there is evidence that there is variation in women's preferences for vaginal lubrication [41], very little is known about rectal lubrication and douching practices in preparation for anal intercourse among women [24]. More research is needed to understand how current routines and practices that are concurrent with anal intercourse will impact whether a product is found acceptable among women and men.

In summary, we collected acceptability data on UC781 gel, provided participants with the opportunity to elaborate on their ratings of the product, and found no serious acceptability problems that would make it advisable to introduce significant changes in the development path for this product. Acceptability research is essential in early phases of microbicide development to aid in understanding user preferences for a product. Making changes to a product earlier rather than later in development will reduce the total cost of development significantly and ultimately achieve the desired outcome, increased use of a product that would decrease the global burden of HIV. However, it is imperative to assess acceptability of this candidate over a longer period of time and concurrent with anal intercourse, as behavioral and contextual factors could shape microbicide acceptability and therefore influence up-take and continued use by individuals [26, 27].

Acknowledgments This research was supported by an IPCP U19 grant from NIAID (\#AI060614). The authors gratefully acknowledge the assistance of the NIAID/DAIDS Program, Medical and Clinical Operations officers, Jim Turpin, Jeanna Piper and Grace Chow and support for this NIAID U19 award. The authors thank Christine Mauck and Henry Gabelnick at CONRAD who were instrumental in completing this trial, as well as Anne-Marie Corner and Biosyn, Inc. who were instructive in the initial preparation and trial launching. The authors also thank William Cumberland at UCLA for his statistical advice on this paper. The authors especially want to thank the participants who took part in this study.

Open Access This article is distributed under the terms of the Creative Commons Attribution Noncommercial License which permits any noncommercial use, distribution, and reproduction in any medium, provided the original author(s) and source are credited.

\section{References}

1. Carballo-Diéguez A, Stein Z, Sáez H, et al. Frequent use of lubricants for anal sex among men who have sex with men: the HIV prevention potential of a microbicidal gel. Am J Public Health. 2000;90(7):1117-21.

2. Mantell JE, Myer L, Carballo-Diéguez A, et al. Microbicide acceptability research: current approaches and future directions. Soc Sci Med. 2005;60:319-30.

3. Rockefeller Foundation. The science of microbicides: accelerating development. A report by the science working group of the microbicide initiative. New York: Rockefeller Foundation; 2002.
4. McGowan I. Rectal microbicides: a new focus for HIV prevention. Sex Transm Infect. 2008;84:413-7.

5. Gross M, Buchbinder SP, Celum C, et al. Rectal microbicides for U.S. gay men. Are clinical trials needed? Are they feasible? HIVNET vaccine preparedness study protocol team. Sex Transm Dis. 1998;25:296-302.

6. Baldwin JI, Baldwin JD. Heterosexual anal intercourse: an understudied, high-risk sexual behavior. Arch Sex Behav. 2000; 29:357-73.

7. Catania J, Canchola J, Binson D, et al. National trends in condom use among at-risk heterosexuals in the United States. JAIDS. 2001;27:176-82.

8. Erickson PI, Bastani R, Maxwell AE, et al. Prevalence of anal sex among heterosexuals in California and its relationship to other AIDS risk behaviors. AIDS Educ Prev. 1995;7:477-93.

9. Flannery D, Ellingson L, Votaw KS, Schaefer EA. Anal intercourse and sexual risk factors among college women, 1993-2000. Am J Health Behav. 2003;27(3):228-34.

10. Friedman SR, Flom PL, Kottiri BJ, et al. Prevalence and correlates of anal sex with men among young adult women in an inner city minority neighborhood. AIDS. 2001;15(15):2057-60.

11. Gorbach PM, Manhart LE, Hess KL, Stoner BP, Matin DH, Holmes KK. Anal intercourse among young heterosexuals in three sexually transmitted disease clinics in the United States. Sex Transm Dis. 2009;36(4):193-8.

12. Gross M, Holte SE, Marmor M, Mwatha A, Koblin BA, Mayer $\mathrm{KH}$. Anal sex among HIV-seronegative women at high risk of HIV exposure. The HIVNET vaccine preparedness study 2 protocol team. JAIDS. 2000;24:393-8.

13. Halperin DT. Heterosexual anal intercourse: prevalence, cultural factors, and HIV infection and other health risks, part I. AIDS Patient Care STDS. 1999;13(12):717-30.

14. Hobfoll SE, Jackson AP, Lavin J, Britton PJ, Shepherd JB. Safer sex knowledge, behavior, and attitudes of inner-city women. Health Psychol. 1993;12(6):481-8.

15. Koblin BA, Chesney MA, Husnik MJ, et al. High-risk behaviors among men who have sex with men in 6 US cities: baseline data from the EXPLORE Study. Am J Public Health. 2003;93:926-32.

16. Tian LH, Peterman TA, Tao G, et al. Heterosexual anal sex activity in the year after an STD clinic visit. Sex Transm Dis. 2008;35(11):905-9.

17. Misegades L, Page-Shafer K, Halperin D, McFarland W, YWS Study Investigators' Group Young Women's Survey. Anal intercourse among young low-income women in California: an overlooked risk factor for HIV? AIDS. 2001;15(4):534-5.

18. Mosher WD, Chandra A, Jones J. Sexual behavior and selected health measures: Men and women 15-44 years of age, United States, 2002. Advance data from vital and health statistics; no 362. Hyattsville: National Center for Health Statistics; 2005.

19. Gray RH, Wawer MJ, Brookmeyer R, et al. Probability of HIV-1 transmission per coital act in monogamous heterosexual, HIV-1 discordant couples in Rakai, Uganda. Lancet. 2001;357:1149-53.

20. Varghese B, Maher JE, Peterman TA, Branson BM, Steketee RW. Reducing the risk of sexual HIV transmission: quantifying the per-act risk for HIV on the basis of choice of partner, sex act, and condom use. Sex Transm Dis. 2002;29(1):38-43.

21. Vittinghoff E, Douglas J, Judson F, McKirnan D, MacQueen K, Buchbinder SP. Per-contact risk of human immunodeficiency virus transmission between male sexual partners. Am J Epidemiol. 1999;150(3):306-11.

22. Carballo-Diéguez A, Dolezal C, Bauermeister JA, et al. Preference for gel over suppository as delivery vehicle for a rectal microbicide: results of a randomised, crossover acceptability trial among men who have sex with men. Sex Transm Infect. 2008;84(6):483-7.

23. Carballo-Diéguez A, Exner T, Dolezal C, Pickard R, Lin P, Mayer KH. Rectal microbicide acceptability: results of a volume escalation trial. Sex Transm Dis. 2007;34:224-9. 
24. Exner TM, Correale J, Carballo-Diéguez A, et al. Women's anal sex practices: Implications for formulation and promotion of a rectal microbicide. AIDS Educ Prev. 2008;20(2):148-59.

25. Gross M, Celum C, Tabet S, et al. Acceptability of a bioadhesive nonoxynol-9 gel delivered by an applicator as a rectal microbicide. Sex Transm Dis. 1999;26:572-8.

26. Morrow KM, Ruiz MS. Assessing microbicide acceptability: a comprehensive and integrated approach. AIDS Behav. 2008; 12(2):272-83.

27. Severy LJ, Newcomer S. Critical issues in contraceptive and STI acceptability research. J Soc Issues. 2005;61(1):45-64.

28. Marks G, Mansergh G, Crepaz N, Murphy S, Miller LC, Appleby PR. Future HIV prevention options for men who have sex with men: intention to use a potential microbicide during anal intercourse. AIDS Behav. 2000;4(3):279-87.

29. Rader M, Marks G, Mansergh G, et al. Preferences about the characteristics of future HIV prevention products among men who have sex with men. AIDS Educ Prev. 2001;13(2):149-59.

30. Carballo-Diéguez A, O'Sullivan LF, Lin P, et al. Awareness and attitudes regarding microbicides and nonoxynol- 9 use in a probability sample of gay men. AIDS Behav. 2006;11:271-6.

31. Tabet SR, Surawicz C, Horton S, et al. Safety and toxicity of Nonoxynol-9 gel as a rectal microbicide. Sex Transm Dis. 1999;26(10):564-71.

32. Celum C. Evaluation of safety and toxicity of rectal microbicides: lessons learned from the first phase I trial of N-9 for rectal use. AIDS. 2001;15(S1):S46.

33. Phillips DM, Taylor CL, Zacharaopoulos VR, et al. Nonoxynol-9 causes rapid exfoliation of sheets of rectal epithelium. Contraception. 2000;62:149-54.
34. Phillips DM, Sudol KM, Taylor CL, et al. Lubricants containing N-9 may enhance rectal transmission of HIV and other STIs. Contraception. 2004;70:107-10.

35. Balzarini J, Naesens L, Verbeken E, et al. Preclinical studies on thiocarboxanilide UC-781 as a virucidal agent. AIDS. 1998; 12:1129-38.

36. Anton P, Adler A, Khanukhova E, et al. A phase 1 rectal safety and acceptability study of UC781 microbicide gel. Conference on Retroviruses and Opportunistic Infections. Montreal, 2009 [abstract 1066].

37. Schwartz JL, Kovalevsky G, Lai JJ, et al. A randomized six-day safety study of an antiretroviral microbicide candidate UC781, a non-nucleoside reverse transcriptase inhibitor. Sex Transm Dis. 1998;35:414-9.

38. Branche V, Cohen JA, Cochon L, Alvarez F. Evaluating the clinical safety of three vaginal applicators: a pilot study conducted in the Dominican Republic. Contraception. 2006;73:72-7.

39. Nodin N, Carballo-Diéguez A, Ventuneac AM, Balan IC, Remien R. Knowledge and acceptability of alternative HIV prevention bio-medical products among MSM who bareback. AIDS Care. 2008;20(1):106-15.

40. Carballo-Diéguez A, Bauermeister JA, Ventuneac A, Dolezal C, Balan I, Remien RH. The use of rectal douches among HIVuninfected and infected men who have unprotected receptive anal intercourse: implications for rectal microbicides. AIDS Behav. 2008;12:860-6.

41. Braunstein S, Van De Wijgert J. Preferences and practices related to vaginal lubrication: implications for microbicide acceptability and clinical testing. J Womens Health. 2005;14(5):424-33. 